\title{
Subsequent Pregnancy after Preterm Prelabor Rupture of Membranes before 27 Weeks' Gestation
}

Jantien L. van der Heyden, MD ${ }^{1}$ Sander M. J. van Kuijk, MSc ${ }^{1,2}$ David P. van der Ham, MD ${ }^{3}$ Kim J. B. Notten, $\mathrm{MD}^{4}$ Timothy Janssen, $\mathrm{MB}^{5}$ Jan G. Nijhuis, MD, $\mathrm{PhD}^{1}$ Christine Willekes, MD, $\mathrm{PhD}^{1}$ Martina Porath, MD, $\mathrm{PhD}^{6}$ Joris A. van der Post, MD, $\mathrm{PhD}^{5}$ Feico Halbertsma, MD, $\mathrm{PhD}^{7}$ Eva Pajkrt, MD, PhD ${ }^{5}$ Ben Willem J. Mol, MD, $\mathrm{PhD}^{5}$

${ }^{1}$ Department of Obstetrics and Gynecology, Maastricht University Medical Center, GROW School for Oncology and Developmental Biology, The Netherlands

2 Department of Epidemiology, Maastricht University Medical Center, The Netherlands

${ }^{3}$ Department of Obstetrics and Gynecology, Martini Hospital, Groningen, The Netherlands

${ }^{4}$ Department of Obstetrics and Gynecology, VieCuri Medical Center, Venlo, The Netherlands

${ }^{5}$ Department of Obstetrics and Gynecology, Academic Medical Center, Amsterdam, The Netherlands

${ }^{6}$ Department of Obstetrics and Gynecology, Máxima Medical Center Veldhoven, The Netherlands

7 Department of Neonatology, Máxima Medical Center Veldhoven, The Netherlands

Am J Perinatol Rep 2013;3:113-118.

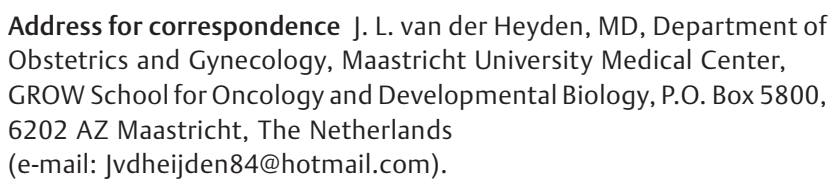

Address for correspondence J. L. van der Heyden, MD, Department of Obstetrics and Gynecology, Maastricht University Medical Center, GROW School for Oncology and Developmental Biology, P.O. Box 5800, 6202 AZ Maastricht, The Netherlands (e-mail: Jvdheijden84@hotmail.com).

\begin{abstract}
Keywords

- preterm rupture of membranes

- recurrence

- preterm birth

Objective Midtrimester preterm prelabor rupture of membranes (PPROM) has a high rate of neonatal mortality and morbidity. The aim of this study was to study outcomes of subsequent pregnancies after a pregnancy with PPROM before 27 weeks' gestation.

Study Design Retrospective study of subsequent pregnancies of women who suffered PPROM before 27 weeks' gestation from 1994 to 2009. The main outcome measure was the risk of recurrence of PPROM before 27 weeks' gestation. The authors also studied preterm birth and pregnancy outcome in the subsequent pregnancy. Finally, they assessed associative factors for subsequent premature delivery.

Results They identified 307 patients, of whom 118 women had a subsequent pregnancy. Of 99 women with complete outcome data, 9 women (9\%) had PPROM before 27 weeks' gestation in a subsequent pregnancy and 35 women (35\%) had a preterm delivery. In 58 (59\%) of pregnancies no major complications occurred. They found three associative factors for premature delivery in a subsequent pregnancy: negative vaginal culture for Group B streptococcus, increasing maternal age and early gestational age at PPROM in the index pregnancy.

Conclusions Women with PPROM before 27 weeks have a 9\% recurrence risk of early PPROM and a risk of $35 \%$ of having a preterm delivery in a subsequent pregnancy.
\end{abstract}

received

May 20, 2013

accepted after revision

June 30, 2013

published online

August 21, 2013
DOI http://dx.doi.org/

10.1055/s-0033-1353389. ISSN 2157-6998.
Copyright $\odot 2013$ by Thieme Medical Publishers, Inc., 333 Seventh Avenue, New York, NY 10001, USA. Tel: +1(212) 584-4662.
License terms

(ㄷ) (i) $\ominus$ (\$) 
Preterm birth is a leading cause of neonatal morbidity and mortality. Preterm prelabor rupture of membranes (PPROM) before 37 weeks' gestation complicates $3 \%$ of all pregnancies ${ }^{1}$ and accounts for approximately $30 \%$ of the preterm births. ${ }^{2}$ It is well known that one of the largest risk factors for preterm delivery is a history of preterm delivery. ${ }^{3,4}$

Previous studies on the risk of PPROM recurrence focus on the recurrence of PPROM in general and not specifically on midtrimester PPROM. - Table 1 summarizes the studies that report on the recurrence risk of PPROM. ${ }^{5-7}$

Getahun et $\mathrm{al}^{5}$ studied over 180,000 women; the other two studies $^{6,7}$ had a much smaller population. All these studies concluded that the risk of PPROM recurrence was increased compared with the incidence of PPROM in a general population.

Early PPROM, that is, PPROM before 27 weeks' gestation, occurs in approximately $0.6 \%$ of pregnancies. $^{8}$ Early PPROM is associated with severe complications, such as intrauterine infection, pulmonary hypoplasia, premature birth, contractures, and ultimately neonatal death. ${ }^{2}$ Considering the consequences of early PPROM, women that have had a pregnancy complicated by early PPROM are usually concerned about the recurrence of such a complication.

As most of the data on the recurrence of preterm delivery are not derived from studies addressing early (midtrimester) PPROM the authors aimed to determine the risk of recurrence of early PPROM before 27 weeks' gestation in a subsequent pregnancy. Adequate counseling regarding the risk in a subsequent pregnancy is important to help couples in their consideration of a future pregnancy.

The aim of this study was to assess the risk of PPROM before 27 and between 27 and 34 weeks in patients with a previous pregnancy with PPROM before 27 weeks. The second aim was to identify the recurrence risk of preterm birth in a subsequent pregnancy.

\section{Methods}

The authors performed a retrospective cohort study between 1994 and 2009 in three perinatal centers with a regional function in the Netherlands. These centers were the Máxima Medical Center (MMC) in Veldhoven, the Academic Medical Center (AMC) in Amsterdam, and the Maastricht University Medical Center (MUMC) in Maastricht. Patients were identified from electronic databases. All patients who were diagnosed with preterm prelabor rupture of membranes before 27 weeks' gestation in the index pregnancy were included in the study. Demographic, medical, and obstetric data were obtained from medical files. Information on subsequent pregnancies was gained from medical files and patients were contacted personally if information in the medical files was not adequate. If patients were lost to follow-up, the authors tried to obtain contact information from other sources (mostly through the general practitioner). A subsequent pregnancy was defined as a singleton or multiple pregnancy, both ongoing pregnancies as miscarriages. Only data on the first subsequent pregnancy after the index pregnancy were used for analysis.

\section{Statistics}

The primary outcome among women with a subsequent pregnancy was recurrence of PPROM before 27 weeks' gestation. Results were expressed as absolute numbers with a percentage, mean with standard deviation, median with interquartile ranges, and odds ratio (OR) with $95 \%$ confidence intervals $(\mathrm{CI})$.

The authors used univariable and multivariable logistic regression to identify potential prediction variables in the index pregnancy. They considered maternal age, gestational age at PPROM (index pregnancy), gestational age at delivery, vaginal culture positive for Group B streptococcus (GBS), live birth in index pregnancy, or postpartum death as potential prediction variables. Live birth was defined as a neonate born with Apgar score after 1 minute $\geq 1$ at any gestational age. Postpartum death was defined as death of a live born neonate at any time after birth. They assessed multiple variables from the index pregnancy in a logistic regression analysis to determine which variables can be used as associative factors for preterm birth in a subsequent

Table 1 Studies reporting on the risk of recurrence of PPROM or preterm birth

\begin{tabular}{|l|l|l|l|l|}
\hline Study & Year & Number of patients & Results & Conclusion \\
\hline Getahun et al $^{5}$ & 2010 & 180,940 women & $\begin{array}{l}\text { Recurrence risk of PPROM among } \\
\text { white women 5.7 and 10.3\% among } \\
\text { African American women }\end{array}$ & $\begin{array}{l}\text { Short interval between index and } \\
\text { subsequent pregnancy is associated } \\
\text { with increased risk for PPROM } \\
\text { recurrence }\end{array}$ \\
\hline${\text { Asrat et } \text { al }^{6}}^{1991}$ & 121 women & $\begin{array}{l}\text { Risk of recurrence of PPROM: } 32.2 \% \\
\text { No association between estimated } \\
\text { GA at time of rupture } \\
\text { in index pregnancy, latency period } \\
\text { and interval between pregnancies }\end{array}$ & $\begin{array}{l}\text { There is a significant risk of } \\
\text { recurrence and need to have } \\
\text { close follow-up in their } \\
\text { subsequent pregnancies }\end{array}$ \\
\hline Lee et al & 2003 & 114 women & $\begin{array}{l}\text { Recurrence rate of PPROM: 16.7\% } \\
\text { Recurrence rate of preterm } \\
\text { delivery: 34.2\% }\end{array}$ & $\begin{array}{l}\text { The risk for recurrent preterm } \\
\text { premature rupture of membranes } \\
\text { is increased by 20-fold and for } \\
\text { recurrent preterm delivery by } \\
\text { almost 4-fold }\end{array}$ \\
\hline
\end{tabular}

Abbreviations: GA, gestational age; PPROM; preterm prelabor rupture of membranes. 


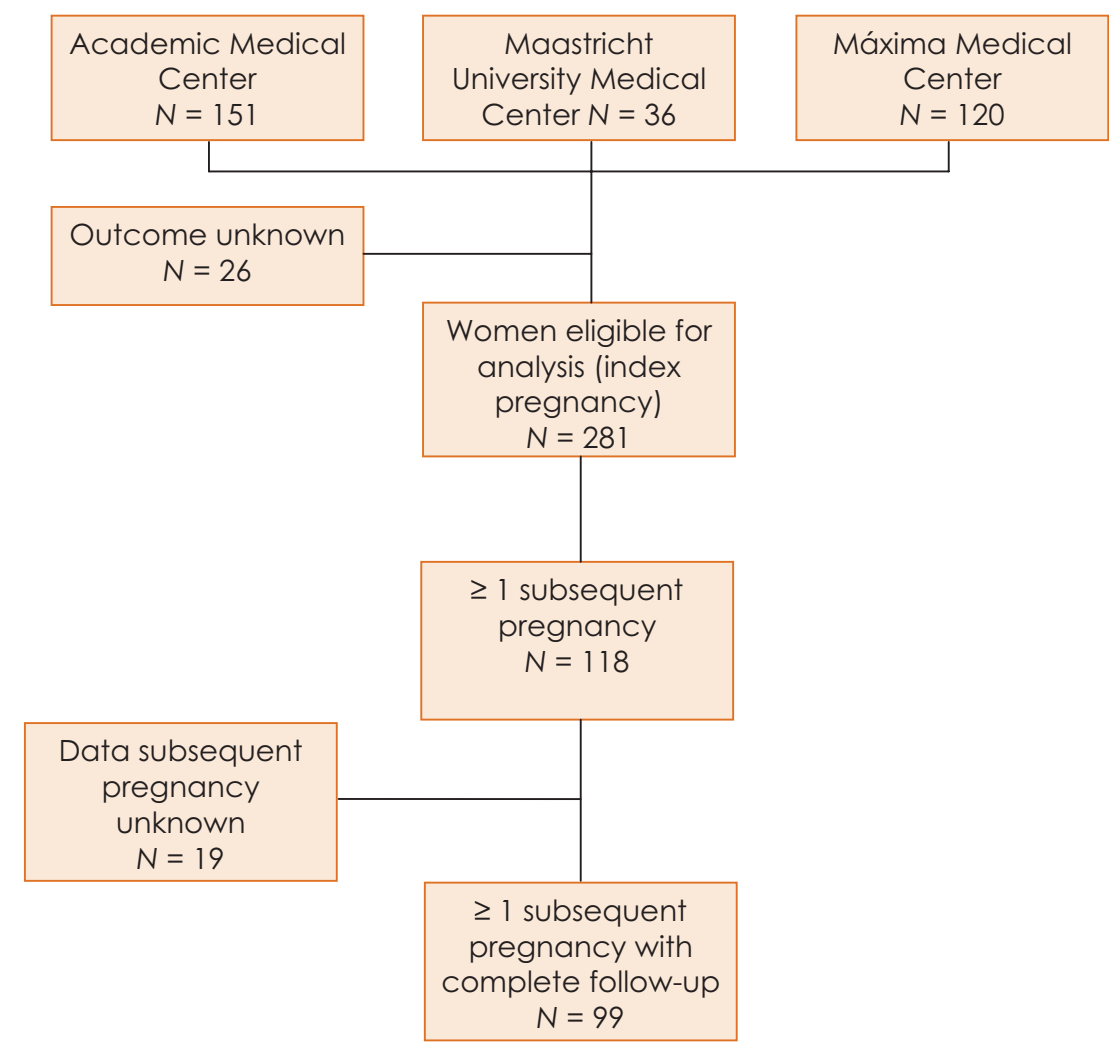

Fig. 1 Flowchart of women included in the study.

pregnancy. Factors with a $p$ value $<0.10$ were considered as factors with associative value.

A Kaplan-Meier curve was constructed to assess time to delivery. All data were analyzed using Excel 2007 (Microsoft Office) and SPSS version 20.0 for Windows (SPSS Inc., Chicago, IL).

\section{Results}

A flowchart of women included in the study is shown in - Fig. 1. The authors identified 307 patients with a singleton pregnancy or multiple pregnancies. Of these patients, 26
(8.5\%) were lost to follow-up, 118 (38\%) had at least one subsequent pregnancy, and 163 (53\%) did not conceive again.

- Table 2 shows the baseline demographic and clinical characteristics and perinatal outcomes of the 118 index pregnancies. In 19 pregnancies data on the subsequent pregnancy were unknown, leaving 99 pregnancies eligible for analysis. In - Table 3, perinatal outcome in the subsequent pregnancy is shown.

The mean gestational age at delivery in the subsequent pregnancy was 35 weeks and 6 days ( $\pm 6.0 \mathrm{~d})$. Overall 9 women (9\%) delivered before 27 weeks, 13 (13\%) between 27 and 34 weeks, 13 (13\%) between 34 and 37 weeks, and

Table 2 Demographic factors index pregnancy

\begin{tabular}{|c|c|c|c|}
\hline Factors & Number $(n=118)$ & Percentage (\%) & Mean (range) [SD] \\
\hline Maternal age & & & $30.2(17.8-44.6)[5.1]$ \\
\hline \multicolumn{4}{|l|}{ Parity } \\
\hline Nulliparity & 63 & 53 & \\
\hline Multiparity & 55 & 47 & \\
\hline Singleton pregnancy & 105 & 89 & \\
\hline \multicolumn{4}{|l|}{ Multiple pregnancies } \\
\hline Twins & 12 & 10 & \\
\hline Triplets & 1 & 0.8 & \\
\hline Gestational age at PPROM & & & $21.9(14.9-26.9)[2.9]$ \\
\hline Vaginal culture positive for GBS & 20 & 17 & \\
\hline
\end{tabular}

Abbreviations: GBS, Group B streptococcus; PPROM; preterm prelabor rupture of membranes. 


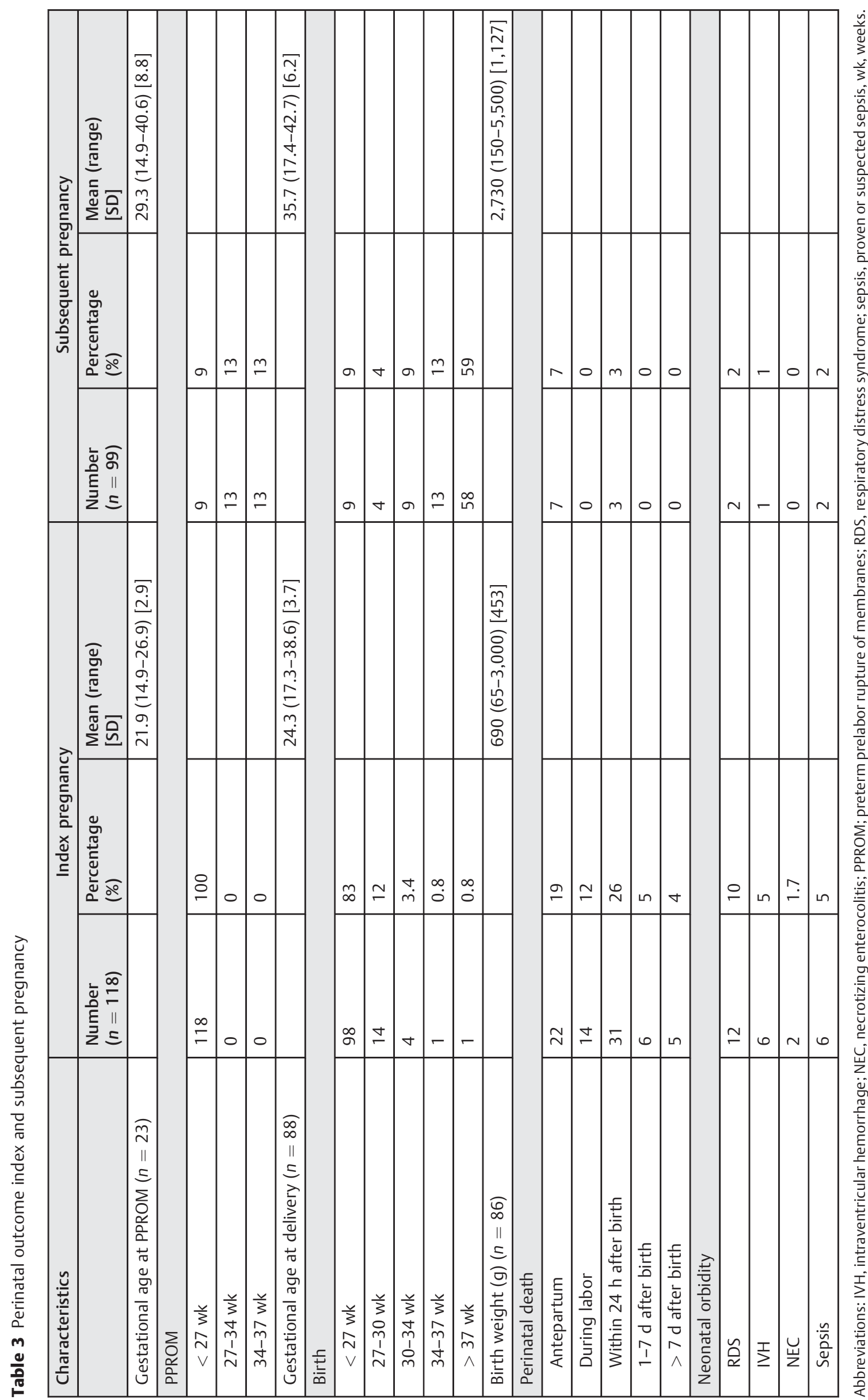




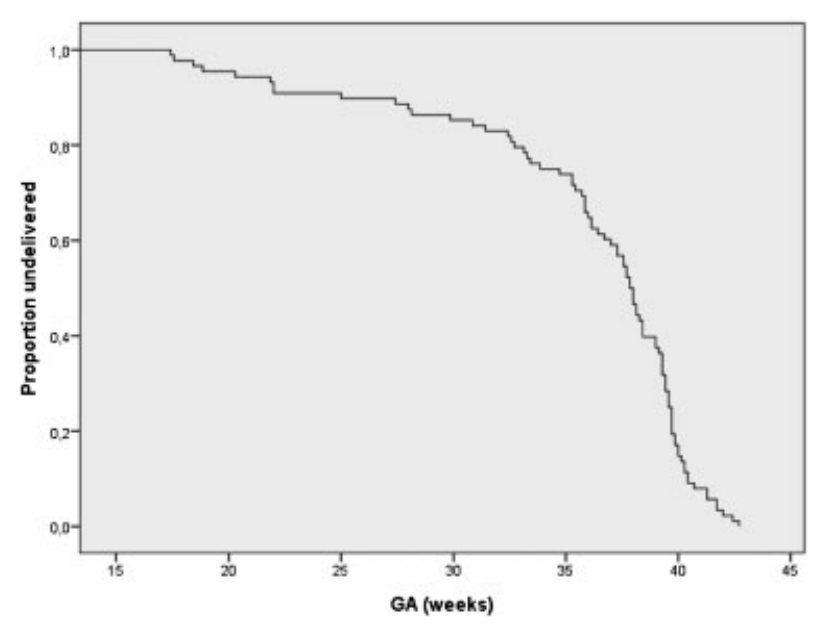

Fig. 2 Kaplan-Meier curve. GA, gestational age.

58 women (59\%) delivered at term. The Kaplan-Meier curve (-Fig. 2) shows that in the subsequent pregnancy, $50 \%$ of women delivered before a gestational age of 37.9 weeks.

Recurrence of PPROM before 27 weeks occurred in nine women (9\%), while six women (6\%) suffered PPROM between 27 and 34 weeks' gestation. In the subsequent pregnancy, there were 71 deliveries after 34 weeks' gestation without major complications (72\%).

- Table 4 shows potential associative factors for preterm delivery in a subsequent pregnancy. In the multivariable analysis, positive vaginal culture for GBS reduced the risk of preterm delivery (OR 0.19, 95\% CI: 0.04-0.91) and (increasing) maternal age (OR 1.12, 95\% CI: 1.0-1.26) was an associative factor for preterm delivery. Moreover, (early) gestational age at PPROM in the index pregnancy, but not gestational age at delivery was also slightly associative for renewed preterm birth (OR 0.97, 95\% CI: 0.94-1.0).

\section{Discussion}

In this retrospective study, the authors studied the risk of recurrence in midtrimester PPROM, since perinatal morbidity and mortality is much higher in this group compared with the group with advanced gestational ages. In this study, the recurrence risk of PPROM before 27 weeks was $9 \%$. A total of $22 \%$ of patients delivered before 34 weeks. Neonatal morbidity is mainly related to the early gestational age at delivery. From that perspective, a recurrence rate of PPROM before 34 weeks of $15 \%$ is relatively high. The results are pretty comparable to data from previous reported studies, with an increased risk of recurrent PPROM of 6 to 32\%. ${ }^{5-7}$

These studies however, reported on PPROM in general and not on midtrimester PPROM. The recurrence risk of premature delivery of $34 \%$ reported by Lee et al is also comparable to the recurrence risk after midtrimester PPROM in this study.

\section{Strengths}

This study included consecutive patients who matched the inclusion criteria. From 307 index pregnancies, only 118 women had a subsequent pregnancy. From this group, the authors could obtain the most important information from 99 pregnancies.

They specifically studied the recurrence risk of PPROM before 27 weeks' gestation, because of the high neonatal mortality and morbidity in this group. This might result in high impact on parents, followed by fear of a renewed pregnancy.

\section{Weaknesses}

Potential drawbacks of the present study are the retrospective nature and the loss to follow-up rate. As the authors could not gain information on $9 \%$ of the pregnancies, and they missed follow-up on another $16 \%$ of the women. The retrospective character hampered complete data collection. Despite these limitations, they believe that their wide time period and the multicenter data collection increased the sample size for this relatively rare disease of PPROM before 27 weeks.

Table 4 Risk factors for preterm delivery before 37 weeks in subsequent pregnancy

\begin{tabular}{|l|l|l|l|l|l|l|}
\hline \multirow{2}{*}{ Clinical characteristic } & \multicolumn{3}{|c|}{ Univariable analysis } & \multicolumn{4}{|c|}{ Multivariable analysis $^{\mathbf{b}}$} \\
\cline { 2 - 7 } & OR & $95 \% \mathrm{Cl}$ & $p$ Value & OR & $95 \%$ CI & $p$ Value \\
\hline Maternal age (y) & 1.07 & $0.98-1.18$ & 0.14 & 1.12 & $1.00-1.26$ & 0.05 \\
\hline GA at PPROM index pregnancy (d) & 0.98 & $0.96-1.00$ & 0.11 & 0.97 & $0.94-1.00$ & 0.09 \\
\hline GA at birth index pregnancy (d) & 0.99 & $0.97-1.00$ & 0.15 & 1.00 & $0.97-1.03$ & 1.00 \\
\hline Positive vaginal culture (GBS) & 0.31 & $0.08-1.22$ & 0.09 & 0.19 & $0.04-0.91$ & 0.04 \\
\hline Life birth & 0.42 & $0.17-1.02$ & 0.06 & 0.51 & $0.10-2.5$ & 0.41 \\
\hline Postpartum death & 1.10 & $0.61-2.1$ & 0.71 & 2.00 & $0.81-4.7$ & 0.14 \\
\hline
\end{tabular}

Abbreviations: Cl, confidence interval; d, days; GA, gestational age; GBS, Group B streptococcus; OR, odds ratio; PPROM; preterm prelabor rupture of membranes; $y$, years.

Note: 88 cases were analyzed.

${ }^{a}$ Number of missing data: 30 .

${ }^{\mathrm{b}}$ Number of missing data: 42 . 
van der Heyden et al.

\section{Possible Predictive Factors}

The authors found that positive vaginal culture for GBS in the index pregnancy decreased the risk of premature delivery in a subsequent pregnancy. This is the opposite of what they expected. They hypothesized that, women with a known positive culture for GBS might be more intensively examined or treated in a subsequent pregnancy. They tried to check this hypothesis using their data. Unfortunately, they have very limited information on the use of antibiotics in the subsequent pregnancy (only known in $36 \%$ of cases). With these limited data, they were not able to confirm this hypothesis. In both groups (use of antibiotics vs. no use of antibiotics), $15 \%$ of women were known GBS positive in the index pregnancy.

A second factor that increased the risk of premature delivery in the subsequent pregnancy was increasing maternal age. The third factor with predictive value is gestational age at PPROM in the index pregnancy. The OR for positive culture for GBS was 0.19 , for maternal age 1.12 , and for gestational age at PPROM 0.97, respectively. Therefore, these factors can be considered as potential associative factors and seem to be clinically relevant predictors.

The importance of this study is that obstetrician gynecologists may use the results to counsel more objectively. Patients should be aware of the higher risk of recurrence to make a proper decision about the continuation of the pregnancy. However, authors were surprised to see that less than $50 \%$ of the women decided to conceive again, while the outcome of the subsequent pregnancy was relatively good. With data from their study, patients are able to make better decisions for future pregnancies.

\section{Conclusion}

Women with PPROM before 27 weeks' gestation in a pregnancy have an increased risk of recurrence of early PPROM. Furthermore, the risk of a premature delivery in future pregnancies is $35 \%$, which is approximately 3 to 4 times higher compared with the risk in a general population, but still allows a new conception after previous early PPROM. The risk of recurrence of PPROM or preterm birth is increased after a previous pregnancy with midtrimester PPROM.

\section{Contribution to Authorship}

All included authors fulfill the requirements for authorship.

\section{Ethics Approval}

Ethical approval was not needed as the retrospective observational character of this study does not require such approval in The Netherlands.

\section{Note}

Poster presentation at the 31st Annual Meeting of the Society for Maternal-Fetal Medicine, held on February 11, 2011 at San Francisco, CA.

\section{Disclosure of Interest}

No conflict of interest related to the findings reported in this article is reported by any of the authors. There were no sources of financial support.

\section{References}

1 Guideline of the Dutch Society for Obstetrics and Gynecology: Rupture of the membranes before the start of labor; Version 1.0 [in Dutch]. http://nvog-documenten.nl/index.php?pagina=/richtlijn/ pagina.php\&fSelectTG_62=75\&fSelectedSub=62\&fSelectedParent=75. Accessed March 9, 2011

2 Ernest JM. Neonatal consequences of preterm PROM. Clin Obstet Gynecol 1998;41(4):827-831

3 Ekwo EE, Gosselink CA, Moawad A. Unfavorable outcome in penultimate pregnancy and premature rupture of membranes in successive pregnancy. Obstet Gynecol 1992;80(2):166-172

4 Heffner LJ, Sherman CB, Speizer FE, Weiss ST. Clinical and environmental predictors of preterm labor. Obstet Gynecol 1993;81(5 Pt 1):750-757

5 Getahun D, Strickland D, Ananth CV, et al. Recurrence of preterm premature rupture of membranes in relation to interval between pregnancies. Am J Obstet Gynecol 2010;202(6):e1-e6

6 Asrat T, Lewis DF, Garite TJ, et al. Rate of recurrence of preterm premature rupture of membranes in consecutive pregnancies. Am J Obstet Gynecol 1991;165(4 Pt 1):1111-1115

7 Lee T, Carpenter MW, Heber WW, Silver HM. Preterm premature rupture of membranes: risks of recurrent complications in the next pregnancy among a population-based sample of gravid women. Am J Obstet Gynecol 2003;188(1):209-213

8 Schucker JL, Mercer BM. Midtrimester premature rupture of the membranes. Semin Perinatol 1996;20(5):389-400 